\title{
Modular Mobile Robotic Kit FOR Prototyping AND DEbugGing of CONTROL AlgorithMS
}

\author{
Oleg Shmakov, Dmitrii Korolev, Dmitrii Popov, \\ Nikolai Kitaev \& Alexei Korotkov
}


This Publication has to be referred as: Shmakov, O[leg]; Korolev, D[mitrii]; Popov, D[mitrii]; Kitaev, N[ikolai] \& Korotkov, A[lexei] (2017). Modular Mobile Robotic Kit for Prototyping and Debugging of Control Algorithms, Proceedings of the 28th DAAAM International Symposium, pp.0950-0956, B. Katalinic (Ed.), Published by DAAAM International, ISBN 978-3-902734-11-2, ISSN 1726-9679, Vienna, Austria

DOI: $10.2507 / 28$ th.daaam.proceedings.132

\begin{abstract}
Rising popularity of robotics in education leads to necessity for modern training programs to have high-quality tool robotic kit. Authors propose a new kit of mobile robotic modules. A modular construction will reduce the time needed for prototyping robotic systems, and will allow to focus directly on tuning control algorithms. In addition, authors focus on the circuit design of the future kit and suggest possible software. The models and structural configurations of wheeled and tracked platforms and manipulator made as basic robotic systems using proposed kit are shown in the article.
\end{abstract}

Keywords: Modular Kit; Mobile Robotic; Prototyping; Algorithmic Debugging

\section{Introduction}

Robotics is an advanced course of science and technology and includes a variety of fields such as construction, modeling, programming, etc. One of the major problems of modern robotics appears to be the development of education programs [1-3]. Today, "sport" approach to the education is generally used; its intention is to prepare projects for national or international competitions and festivals. The teaching process in that case includes learning element base and basic constructions, basics of programming language, solving simple tasks such as path following, obstacle avoidance, etc., iterative preparations of robots due to the regulations of specific competition. However, drawbacks of "sport" robotics are obvious - quick results do not guarantee getting high quality knowledge, as well as, vide variety of competitions do not often allow to make an effective learning process. Opposite to the mentioned approach is a popular paradigm STEM (Science Technology Engineering Mathematics), which presents robotics as an interactive tool, which helps to practice theoretical knowledge about Exact and Natural Sciences [4]. An issue during STEM appliance is to create qualitative and reliable training tools, which could provide essential hardware and software to solve robotics tasks $[3,5]$.

Available on the market robotics kits (LEGO Mindstorms, Fischertechnik, Makeblock, etc. [6-10]) despite their popularity usually have the element base which is insufficient to execute complex algorithms, besides, since they consist of small parts, the major part of learning process is occupied by prototyping instead of studying control tasks. To solve mentioned problems authors of the article offer a kit of mobile robotic modules (MRM). Simple and fast assembly using complete modules will reduce the time needed for prototyping robotic systems and will allow to focus directly on control algorithms implementation. 


\section{Constructions and configurations of a mobile modular robotic kit}

A large modules assembly is used for the realization of the modular kit. According to that method, all modules should be the same size (as Moss and Cubelets have [11, 12]) or connectors should be scalable. Joining of modules and installation on the frame (if there is one) could be done without fasteners, such as screws, nuts, etc., for that purpose modules have quick-detached connectors, such as:

- the universal coupler to fasten drive modules and sensors. That coupler is a combination of rail type mountings on the frame. A module is placed on a rail, and there are options whether to let it to move forward-backward along the rail or to fix it with vices;

- fasteners (thread bushing) to connect drive modules and robot power supply, such as propulsion engines, mechanical transmissions. That joint needs knurled screws. Using that connection type, kit modules could be installed on different frames besides one from MRM kit;

- the universal coupler to transmit torque between drive and mechanical transmissions modules, as well as to connect with locomotion actuators such as wheels, caterpillar tracks, etc. Those connectors are represented as a separate category in order to assure strong connection and the absence of mechanical gaps, using inner system of shaft misalignment elimination. Bodies of modules are connected with nuts on the output and threads on the input of transmissions modules, while an elastomer coupling transmits the moment.

According to suggested kit design, each module is a completely closed system. Regarding the necessary functionality which MRM model should have in order to perform different control algorithms, the following modules were designed:

- CSM - control system module, which the user directly works with and upgrades its software;

- APS - autonomous power supply to support other modules;

- PDM - power drive module, which is motor with control electronics to move around the whole robotic system;

- IM - instrumentation modules, which have different sensors, such as distance, touch, light and other sensors;

- $\mathrm{CM}$ - communication module, which connects a robot with a console (a personal computer);

- MTM - mechanical transmissions modules (connectors between motors, grippers, rotators and different locomotion actuators as wheels modules, track modules and caterpillars themselves), which transmit torque from the drive modules and allow the user to increase or reduce full speed of rotation and the moment of motor output shaft and also to put shaft angularly to the motor or to move it.

Here are the most usual control tasks [13-15] for robotic systems (RS):

- controlling robotic system which moves on the high speed and orients itself using variety of markers (along solid and dashed lines) or obstacles (static and moving) in order to automatically correct its path according to the task (avoidance, collision, stop to avoid collision, etc.);

- centralized or decentralized control of RS group, such as an allocation of the robots; modification of group behavior and priorities of individual assignments, if one of the robots has malfunctions; area reconnaissance. In all that cases (and also for the tasks mentioned before), wheeled robots are most suitable, due to their ability to adapt for changes in the surface;

- RS control in the conditions of rough terrain: overcoming various obstacles, both natural and artificial, using running advantages of a particular device, in that case, tracked robots are suitable because of their passing abilities;

- RS control in order to detect and deliver a particular object: specifying the object's location, selecting among other objects according to the input, grasping the object by the most appropriate way from available options or prediction of a failure, delivery in the particular area, using given reference points, in those cases, usually a manipulator is required.

Regarding mentioned control tasks and in order to show all functions of MRM kit (using relevant sensors and other equipment), three basic RS could be made using the kit - wheeled, tracked and four-step manipulator (see Fig. 1).

Besides, other RS models could be designed based on the mentioned MRM. Actually, variety of constructions, which could be assembled using the kit, is only limited by a number of modules. For example, using several MRM kits, which are intended for several basic wheeled RS, there could be made one platform with six wheels, each of which has two degree of freedom. RS like that not only has good passing abilities but also makes it possible to perform turning maneuvers using each wheel. In addition, PDM can be installed on MTM, using body element, so that a gap between the plane with MTM fastening and the one of wheel contact could be modified. Four assemblies like that and a location sensor allow the platform to keep the same level while moving along rough terrain. If you put two PDM on the short base and equip the third one with "passive" leg, two-wheeled RS could be made. The advantages of that model are the facts that few modules are required and prototyping takes a little time. Finally, two short platforms, connected by rotators, allow RS to rotate about the axis, so that all four wheels always remain in connection with rough terrain. 




Fig. 1. Basic RS using MRM kit

\section{Circuit design of MRM kit}

Following design options are suitable for any MRM kit, regardless of the RS configuration or model. All modules that have electronics are connected with the set of RS-485 wires or directly with CSM. To communicate and control the modules, connected with bus, address formats for data exchange are used. Modules get their addresses automatically according to command of CSM. The order of address assignment is defined in advance and depends on the succession of modules. If places of modules from different MRM kits coincide, the same addresses are guaranteed.

The central control element is CSM. It is suggested that one completed system has no more than one CSM. In the base of a module there is STM32F469 microcontroller with ARM Cortex-M4F core. CSM control is performed by user's programs, loaded using USB port in the body of the module. For that purpose, there are the JTAG debugger and USB-toUART Bridge Controller in the module. CSM supplies connected modules with power while controlling current flow and protecting against overpower, for that purpose it is connected with APS. Operating voltage range of APS is $12 \mathrm{~V}$. Moreover, CSM could be connected with other devices, such as sensors and low-power actuators. For that purpose, modules have some PLT pin connectors with power line of $5 \mathrm{~V}$ and $3.3 \mathrm{~V}$, as well as digital and analog inputs/outputs, protected against polarity reversal and overload

All power drive modules are based on gear motors IG-32PGM and IG-16GM with incremental transducers. Electronics, built in modules, provide three-circuit system, controlling motors by winding current, speed and position of a shaft, so that it is possible to perform control algorithms, based on holding the moment, speed and position.

To perform and implement specific algorithms, installation on your RS of different sensors and payloads may be required, depending on the control task you work with. The minimum set of modules, included in MRM kit, consists of four modules, three of which contain several devices and connect with CSM (which connected with CM) by the bus:

- module1: light, temperature sensors and a microphone;

- module 2: a touch sensor, ultrasonic and infrared distance sensors;

- module 3: a dynamic speaker and a lamp;

- module 4: a camera.

In MRM kit there are two communication modules - one is to process and transmit video from cameras, another - to receive control commands and the telemetry data.

To analyze circuit design of each basic RS model from Fig. 1, we will study each module of the RS separately as an assembly scheme. Four-wheeled basic model (see Fig. 2,3) has battery (APS), control system module (CSM), communication modules (CM) for radio control and video transmission, instrumentation modules (IM), such as a camera, distance sensors and lamps and a manipulator base.

On the base there are power drives (PDM), a gripper (MTM) and a camera (IM). On the lower part of platform there are four propulsion engines (on the PDM and MTM base). There are two fenders, to which you can fasten IM, above the wheels. Thus, using MRM kit, RS with four-wheeled drive and expandable functionality could be designed. 


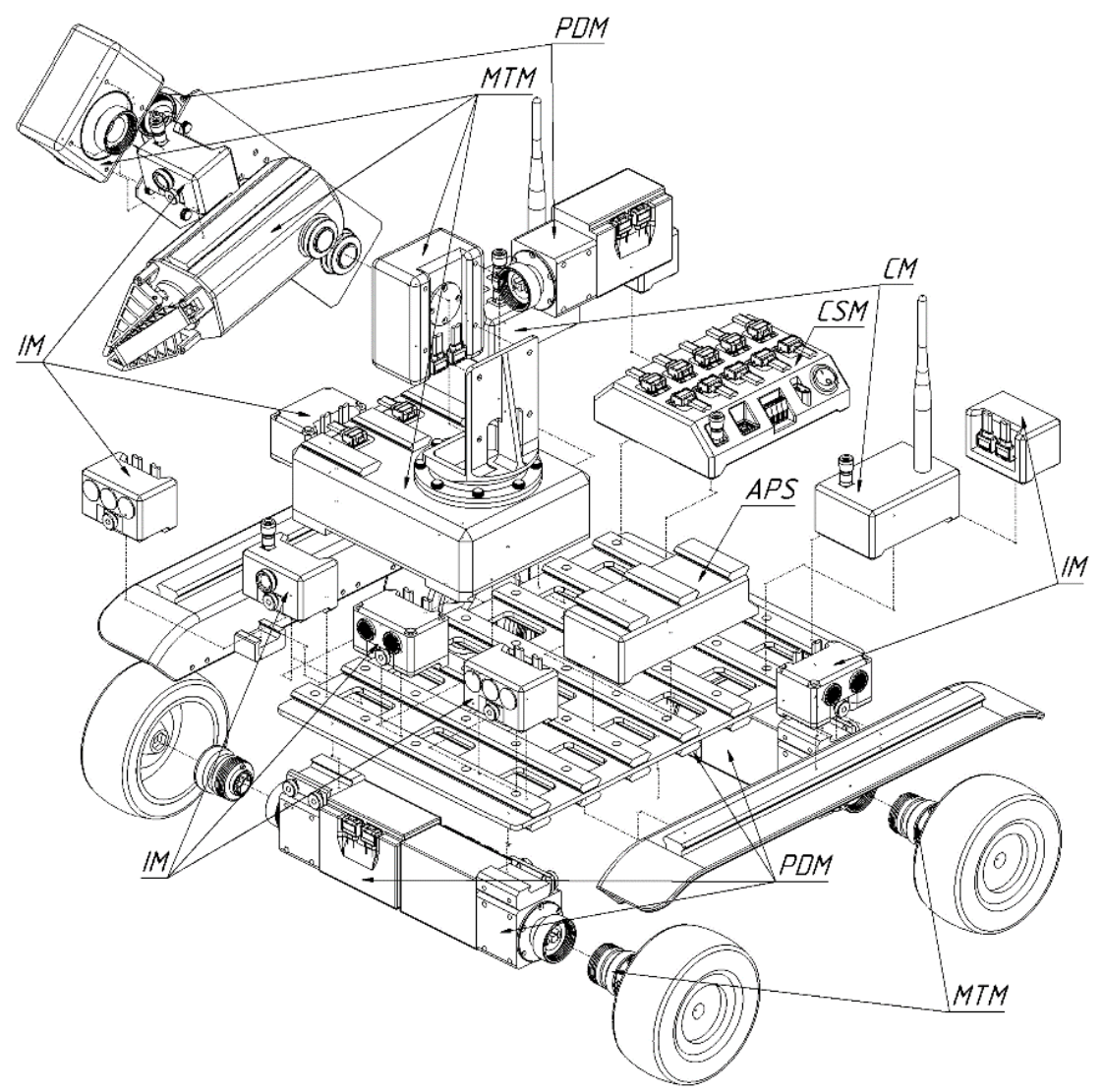

Fig. 2. The assembly scheme of basic four-wheeled RS

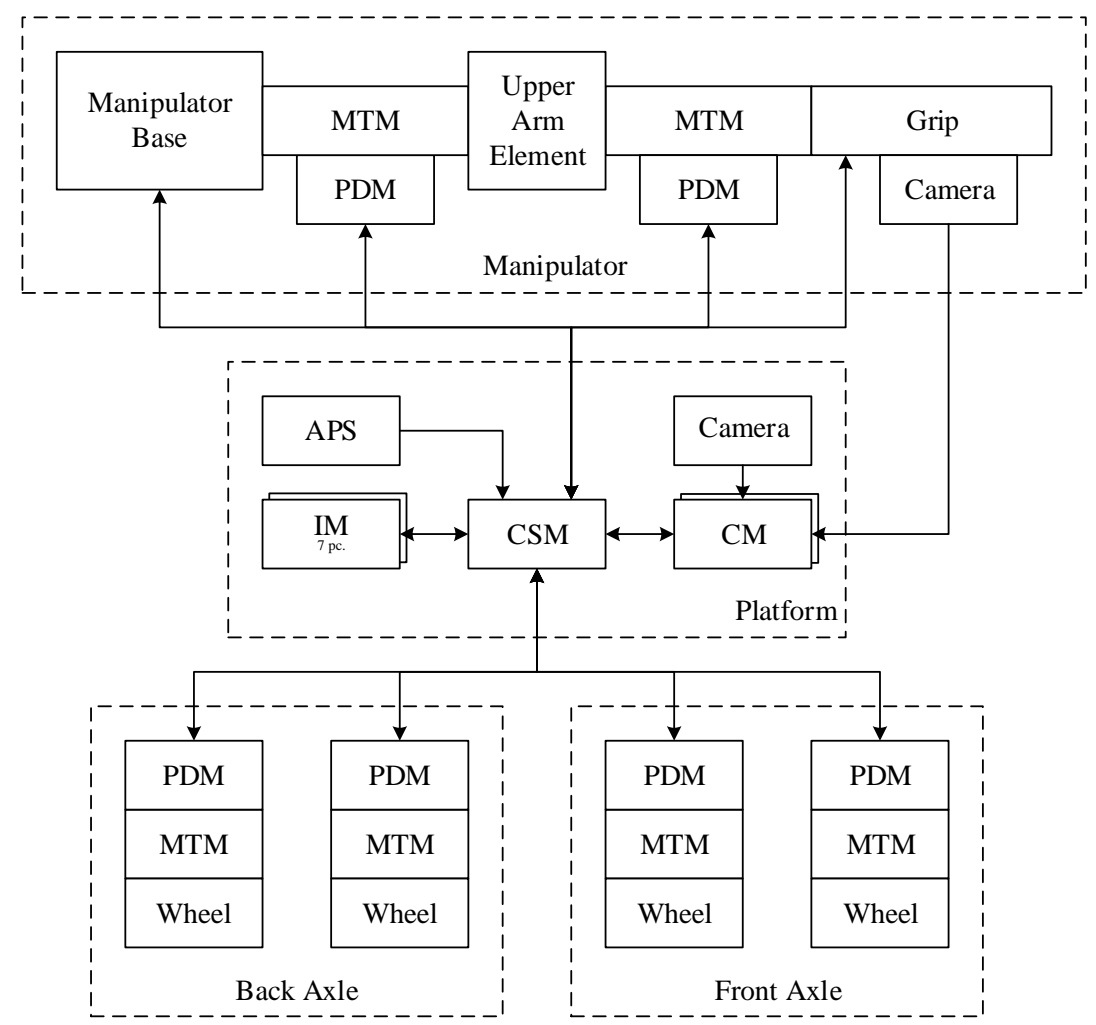

Fig. 3. The structure of four-wheeled RS

Considering applied modules, there is a slight difference between tracked model (see Fig. 4, 5) and the wheeled RS: locomotion actuators are tracks; there are only two propulsion engines (PDM), and each has one cylindrical transmission (MTM); instead of manipulator there is a two-step camera orientation module (MTM). 


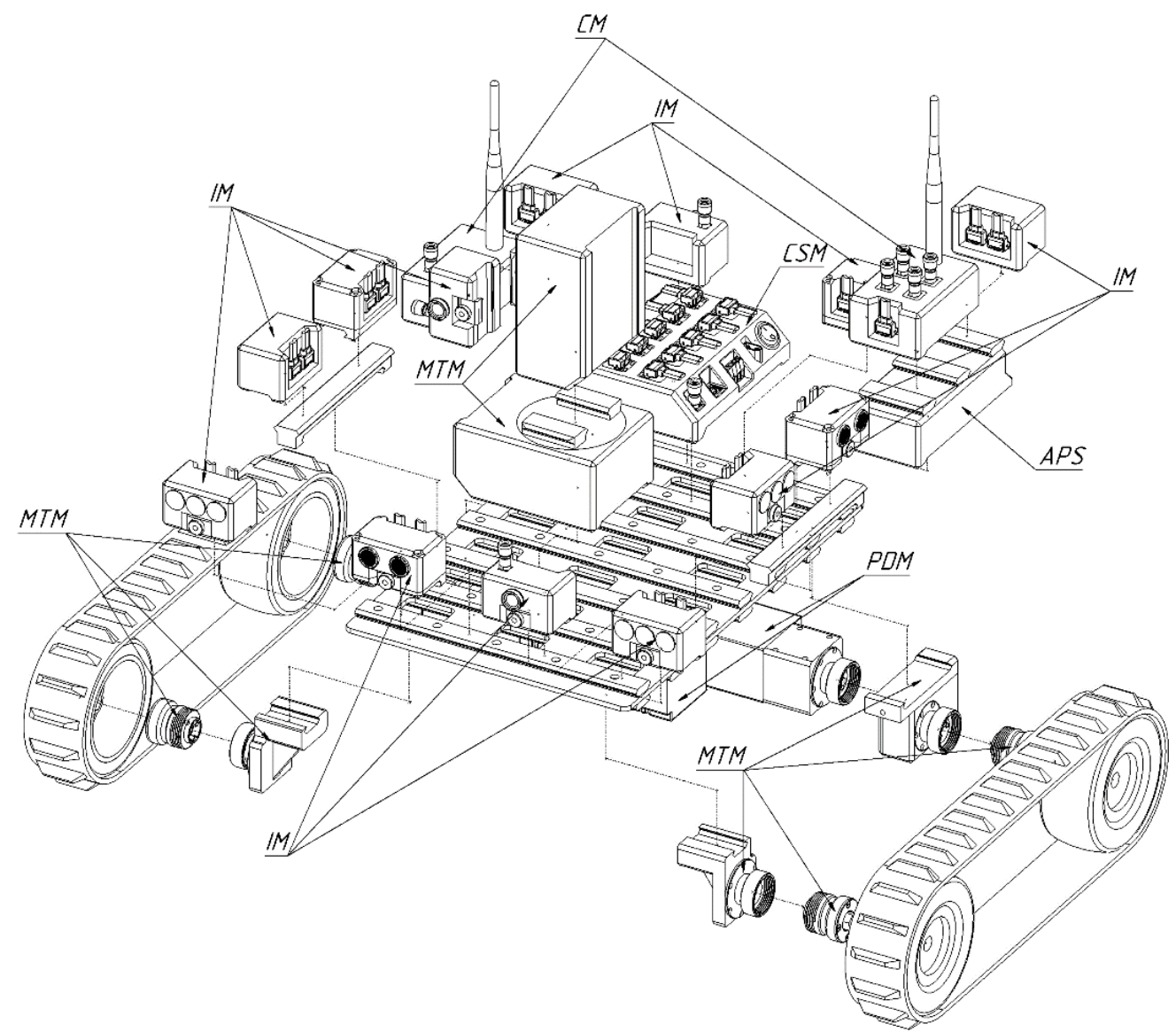

Fig. 4. The assembly scheme of basic tracked RS

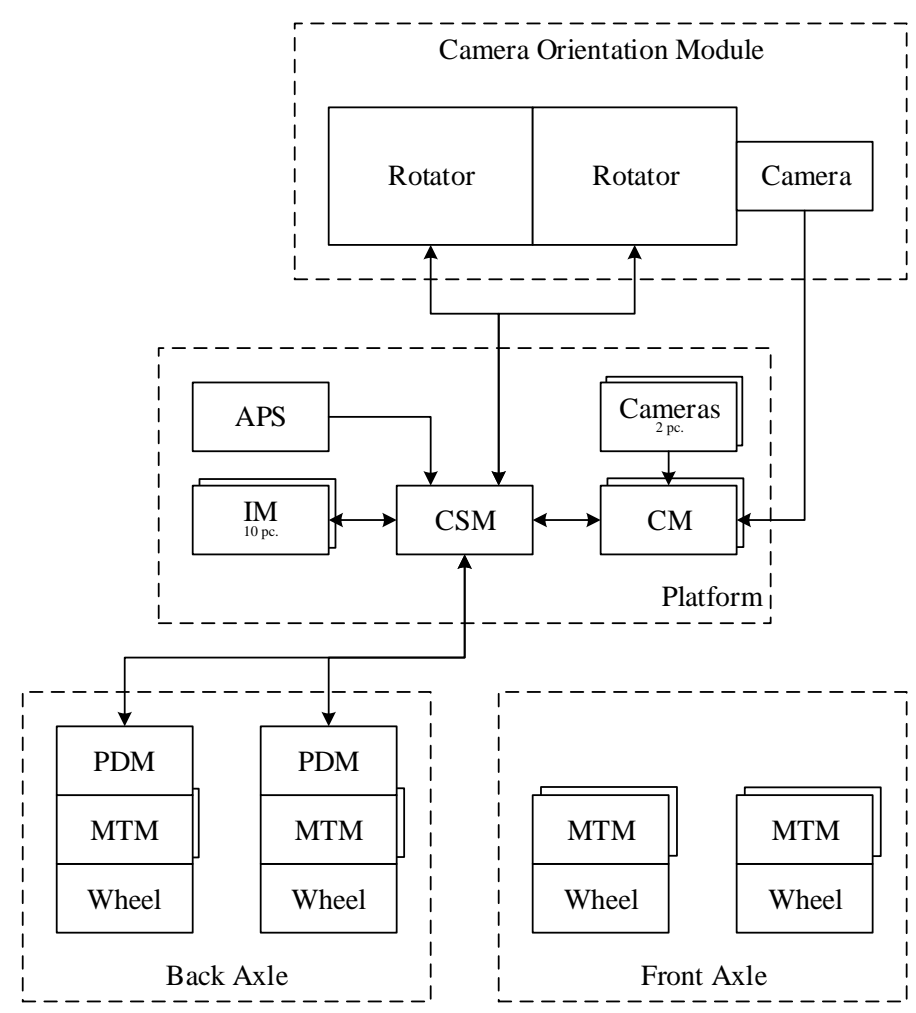

Fig. 5. The structure of tracked RS

Basic model of four-step manipulator with a grip (see Fig. 6, 7) consists of the same modules as the former two, but it also has special transitional body parts. 


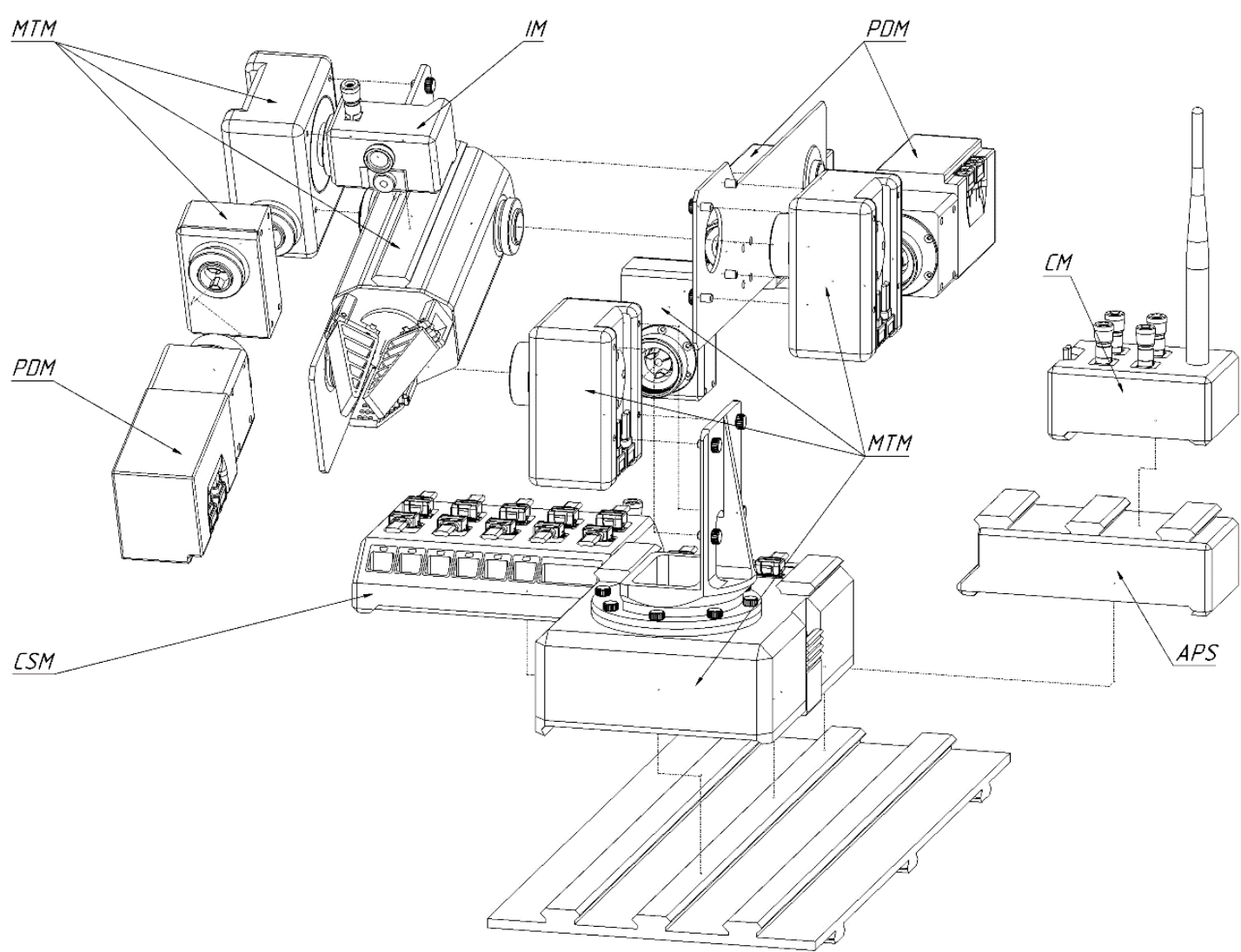

Fig. 6. The assembly scheme of basic RS with four-step manipulator

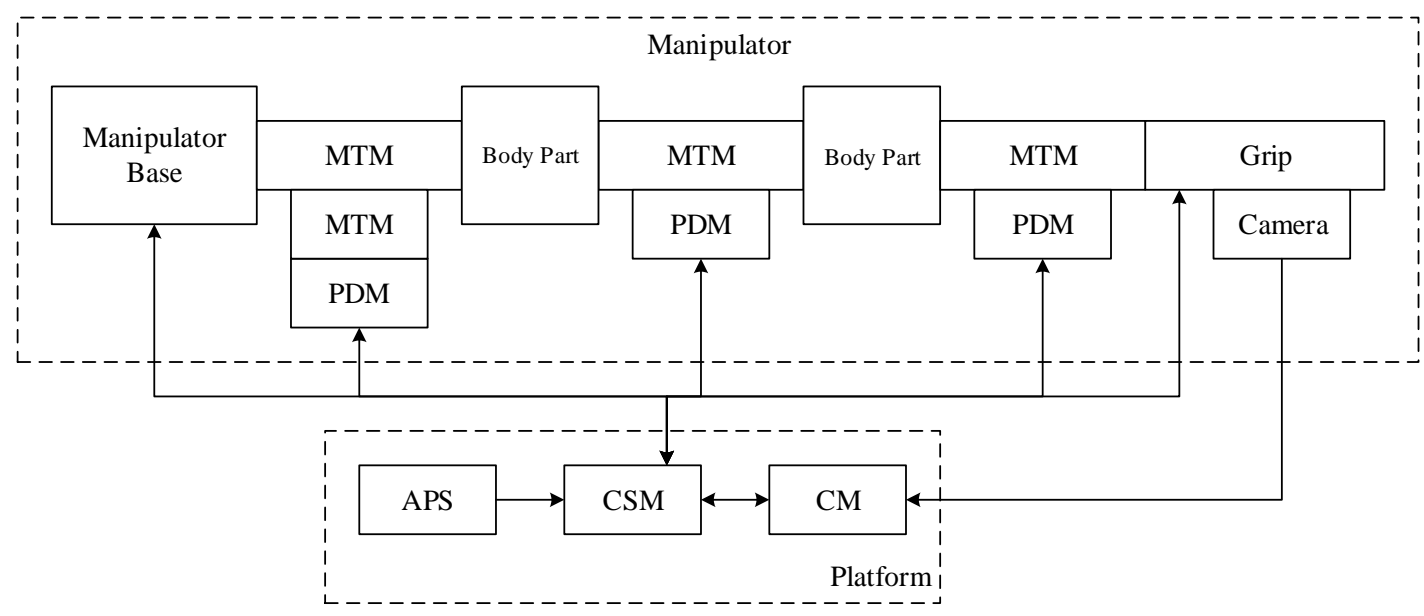

Fig. 7. The structure of RS with four-step manipulator

\section{Software solution of MRM kit}

Now, the authors do not have final decision on the development environment to debug control algorithms. Still, for convenience and complete understanding of MRM kit there are key aspects for further development below. So that the user can easily begin software development, there are two courses of the development environment:

- programming using textual modeling language;

- programming using graphical modeling language.

Textual modeling involves writing source code of a program using keyboard and presumably in $\mathrm{C}$ and $\mathrm{C}++$ languages. For graphical modeling, instead of source code of a program, there are graphical annotations. They could represent whole blocks (lines) of source code and higher abstractions (behavior, module). In view of the successful implementation of a visual development environment for the cybernetic controller TRIK [16], the authors consider the possibility of TRIK STUDIO integration in the software. 


\section{Conclusion}

The main problem of modern educational robotics is to create such kit, which will be useful both in elementary and in middle schools to teach children the basics of engineering and robotics and in high schools and universities for executing and implementing control algorithms. The authors successfully solved this problem by developing MRM kit, the main concept of which was based on the combination of the advantages of a large modular assembly (simplicity and quickness) and the equipment like a powerful controller and a variety of high-quality sensors, which are essential for testing modern algorithms. Moreover, suggested solution could be an effective tool to design and model mobile robotic platforms and industrial robotic complexes for qualified specialists in the research centers and enterprises. Future plans include the production of MRM kit and development of software solutions.

\section{Acknowledgements}

This research is supported by the Ministry of Education and Science of Russia (an agreement № 14.578.21.0124 on granting for the implementation of applied scientific research and experimental development). Unique identificator of ASRED - RFMEFI57815X0124.

\section{References}

[1] http://raor.ru, (2016). Official web site of Russian association of educational robotics, Accessed on: 2016-10-31

[2] Pryanichnikov, V.; Andreev, V.; Bobrov, P.; Biryukova, E.; Frolov, A.; Kharin, K.; Kirsanov, K.; Kostin, A.; Kuvshinov, S.; Marzanov, Y. \& Prysev, E. (2012). Scientific-educational distributed laboratory-software and hardware means. Annals of DAAAM for 2012 \& Proceedings of the 23rd International DAAAM Symposium, Vol. 23, No. 1, pp. 1175-1178, ISSN 2304-1382

[3] Seiler, S.; Ptasik, D. \& Sell, R. (2011). Remote and virtual labs in a distance learning environment. Annals of DAAAM for 2011 \& Proceedings of the 22nd International DAAAM Symposium, Vol. 22, No. 1, pp. 611-612, ISSN 1726-9679

[4] Alvarez, I. (2011). VEX Robotics: STEM Program and Robotics Competition Expansion into Europe, In: Research and Education in Robotics - EUROBOT 2011, Obdrzalek, D. \& Gottscheber, A., pp. 10-16, Springer, ISBN 978-3642-21975-7

[5] Filippov, S.A. (2013). Robotics for children and parents, rus: Robototekhnika dlya detey i roditeley, Nauka, ISBN 978-5-02-038-200-8, St. Petersburg, Russia

[6] Baichtal, J. (2015). Hacking Your LEGO Mindstorms EV3 Kit, Que Publishing, ISBN-13: 978-0-7897-5538-4

[7] Ferrari, M. \& Ferrari, G. (2011). Building Robots with LEGO Mindstorms NXT, Syngress, ISBN-13: 9781597491525

[8] Cuéllar, M.P. \& Pegalajar, M.C. (2014). Design and implementation of intelligent systems with LEGO Mindstorms for undergraduate computer engineers. Computer Applications in Engineering Education, No. 22, pp. 153-166, ISSN 1099-0542

[9] Beliovskaya, L.G. \& Beliovskiy, A.Ye. (2013). Programming microcomputer NXT with LabVIEW, rus: Programmiruyem mikrokompyuter NXT v LabVIEW, DMK Press, ISBN 978-5-94074-594-5, Moscow, Russia

[10] Bagnall, B. (2012). Maximum LEGO NXT: Building Robots with Java Brains 3rd, Variant Press, ISBN-13: 9780986832222

[11] Thai, C. N. \& Paulishen, M. (2011). Using robotis bioloid systems for instructional robotics, Proceedings of IEEE Southeastcon, Nashville, USA, 17-20 March 2011, ISSN: 1558-058X, ISBN: 978-1-61284-738-2, pp. 300-306, IEEE, DOI 10.1109/SECON.2011.5752954

[12] http://www.modrobotics.com, (2016). Official web site of kits MOSS and Cubelets, Accessed on: 2016-10-31

[13] Kee, D. (2011). Educational Robotics Primary and Secondary Education. IEEE Robotics \& Automation Magazine, No. 18(4), pp. 16-19, ISSN 1070-9932

[14] Khalamov, V.N. \& Sagritdinova N.A. (2012). Fischertechnik - basics of educational robotics, rus: Fischertechnik osnovy obrazovatelnoy robototekhniki, Vzglyad, ISBN 978-5-93946-191-7, Chelyabinsk, Russia

[15] Correll, N.; Wailes, C. \& Slaby, S. (2014). A One-Hour Curriculum to Engage Middle School Students in Robotics and Computer Science Using, In: Distributed Autonomous Robotic Systems, Ani Hsieh, M. \& Chirikjian, G., pp. 165-176, Springer, ISBN 978-3-642-55146-8

[16] http://www. trikset.com, (2016). Official web site of TRIK project, Accessed on: 2016-10-31 